TITLE:

NUMERICAL METHODS FOR

INVERSE SCATTERING PROBLEMS

IN TWO-DIMENSIONAL SCALAR

FIELD

$\operatorname{AUTHOR}(\mathrm{S})$ :

Onishi, K.

CITATION:

Onishi, K.. NUMERICAL METHODS FOR INVERSE SCATTERING PROBLEMS IN TWO-

DIMENSIONAL SCALAR FIELD. 数理解析研究所講究録 1990, 724: 178-192

ISSUE DATE:

1990-05

URL:

http://hdl.handle.net/2433/101865

RIGHT: 


\title{
NUMERICAL METHODS FOR INVERSE SCATTERING PROBLEMS IN TWO-DIMENSIONAL SCALAR FIELD
}

\author{
大西和 栄 \\ K. Onishi \\ Applied Mathematics Department \\ Fukuoka University \\ Jonan-ku, Fukuoka 814-01, Japan \\ Phone: 092-871-6631
}

\begin{abstract}
.
Methods for the numerical solution of inverse scattering problems for shape identification from the scattering amplitude are presented. The field is governed by two-dimensional Helmholtz equation in exterior domain subject to plane incident waves. The scatterer is assumed either soft or hard infinitely long cylindrical obstacle. Some numerical results of shape identification problems as well as direct problems corresponding to the inverse problems are demonstrated.
\end{abstract}

1. Introduction

Boundary integral equation method is suited to the scattering problem of homogeneous media, for the 
method can not only reduce the exterior boundary value problem with the domain extending to infinity to a corresponding integral equation on the compact surface of the domain, but also reduce the dimensionality of the problem by one. The Galerkin-collocation method using lower-order finite element interpolation functions has been applied to the numerical solution of the boundary integral equation by the name of Boundary Element Method.

The integral equation method for inverse scattering problems has been investigated by several authors. Among them, Landsberga and Borovikova[1982], Colton and Kress[1983], Kirsch and Kress[1987a, b] presented formulations and some numerical examples for acoustic and electromagnetic waves. Hirose[1987] discussed some problems for elastic waves. To cope with the difficulty of ill-posedness in the inverse problems, methods of regularization are presented in Groetsch[1984], Morozov[1984], and Hofmann[1986] as well.

In this report the author would like to summarize some recent works concerning the numerical solution to inverse scattering problem being done in his office. The problem consists of finding the shape of the smooth obstacle based on the observation of scattering amplitude ( far-field pattern) of acoustic waves of low frequencies. If the far-field pattern in all directions is known exactly, the problem will be reduced to a mathematical minimization problem. If the far-field pattern is known only in a finite number of directions, we shall also consider the restoration of the pattern in the rest of directions as one of the moment problems. We shall also consider a special case when some Fourier components of the far-field pattern are given. Before embarking the solution of inverse problems, one is required to be able to resolve the direct problems satisfactorily. Numerical examples in direct scattering problem are presented, including scattering from circular cylindrical obstacles, numerical demonstration of the Fraunhofer diffraction. A simple numerical example in inverse problem is presented for the shape identification based on the exact knowledge of far-field pattern corresponding to a soft scatterer.

\section{Inverse Scattering Problems}

We shall first describe direct scattering problem rather than we start the problem statements with the description of the inverse problem. Let $\Omega$ be a simply connected bounded domain enclosed by the boundary $\Gamma$ in two dimensional Euclidean space with the coordinates $x=\left(x_{1}, x_{2}\right)$. We may think of $\Omega$ as the cross section of an infinitely long cylindrical scatterer. We assume that $\Gamma$ is smooth of the class $C^{2}$. By $\Omega e$ we shall denote the exterior domain to $\Omega$.

The scattering to be considered in this paper is described by the spatial form $u(x)$ of the scalar wave

$$
u(x)=u^{i}(x)+u^{s}(x), \quad x \in \bar{\Omega}^{e}
$$

where $u i$ denotes the incident plane wave of the form

$$
u^{i}=e^{i k\left(d_{1} x_{1}+d_{2} x_{2}\right)}
$$

with the wave number $k$, the incident direction $\left(d_{1}, d_{2}\right)$, and us the scattered wave. The scattered wave has to satisfy the Helmholtz equation

$$
\left(\Delta+k^{2}\right) u^{s}(x)=0 \text { in } \Omega^{e} .
$$

The Sommerfeld radiation condition in two-dimensional case, which inhibits the reflection of waves from the infinity, is imposed as

$$
\frac{\partial u^{s}}{\partial r}-i k u^{s}=o\left(\frac{1}{\sqrt{r}}\right) \text { as } \quad r=|x| \rightarrow \infty
$$

uniformly with respect to the angle $\theta=\arctan \left(x_{2} / x_{1}\right)$. 
If $\Omega$ is a soft obstacle, the boundary condition is given in terms of the total wave $u$ by the homogeneous Dirichlet condition

$$
u=0 \text { on } \Gamma \text {. }
$$

If $\Omega$ is a hard obstacle, the corresponding boundary condition is the homogeneous Neumann condition

$$
\frac{\partial u}{\partial v}=0 \text { on } \Gamma \text {, }
$$

where $v$ denotes the exterior unit normal to $\Gamma$. The direct problem is to find us in $C^{2}(\Omega e) \cap C(\Omega e \cup \Gamma)$ satisfying (1)-(6) for given ui.

Both the exterior Dirichlet and Neumann problems of the Helmholtz equation with the radiation condition are uniquely solvable. The solution us has the integral representation

$$
u^{s}(\xi)=\int_{\Gamma}\left\{u^{s}(x) \frac{\partial G}{\partial v(x)}(x, \xi)-\frac{\partial u^{s}}{\partial v}(x) G(x, \xi)\right\} d \Gamma(x), \quad \xi \in \Omega^{e}
$$

with the fundamental solution $G$ to the operator $\Delta+k^{2}$ :

$$
\begin{aligned}
& \left(\Delta+k^{2}\right) G(x, \xi)=-\delta(\xi), \\
& G(x, \xi)=\frac{i}{4} H_{0}^{(1)}(k|x-\xi|),
\end{aligned}
$$

where $H_{0}(1)$ is the Hankel function of the first kind of order zero satisfying the radiation condition (4). According to Kress[1989], we know that the scattered wave has an asymptotic expansion of the form

$$
u^{s}(\xi)=\sum_{n=-\infty}^{+\infty} a_{n} H_{n}^{(1)}(k r) e^{i n \theta} \quad \text { as } \quad r \rightarrow \infty
$$

with $\xi=(r, \theta)$ and the constants an.

The scattered wave has the asymptotic form

$$
u^{s}(\xi)=\frac{e^{i k r}}{\sqrt{r}}\left\{f(\theta)+O\left(\frac{1}{r}\right)\right\} \quad \text { as } \quad r \rightarrow \infty
$$

where $f(\theta)$ is the scattering amplitude or far-field pattern of the scattered wave. The scattering cross section is defined by $\sigma(\theta)=4|f(\theta)|^{2 / k}$. We notice that the far-field pattern can be expanded in the Fourier series

$$
f(\theta)=\sum_{n=-\infty}^{+\infty} b_{n} e^{i n \theta}
$$

with

$$
b_{n}=a_{n} \sqrt{\frac{2}{\pi k}} e^{-\frac{2 n+1}{4} \pi i}
$$

This shows that $f(\theta)=0$ implies $u s=0$; one-to-one correspondence between solutions (10) to the Helmholtz equations satisfying the radiation condition and their far-field patterns $f(\theta)$.

In terms of the total wave, the representation (7) has the alternative form

$$
u(\xi)=u^{i}(\xi)+\int_{\Gamma}\left(u \frac{\partial G}{\partial \nu}-\frac{\partial u}{\partial v} G\right) d \Gamma, \quad \xi \in \Omega^{e} .
$$

In the limit as the point $\xi$ tends to a point on the boundary, we can see 


$$
\frac{\Theta(\xi)}{2 \pi} u(\xi)=u^{i}(\xi)+\int_{\Gamma}\left(u \frac{\partial G}{\partial v}-\frac{\partial u}{\partial v} G\right) d \Gamma, \quad \xi \in \Gamma
$$

where $\Theta(\xi)$ denotes the exterior planar angle to $\Omega$ at the boundary point $\xi$. For a smooth $\Gamma$, we have $\Theta(\xi)=\pi$. The equation (15) is the boundary integral equation corresponding to the exterior problem of the Helmholtz equation (3).

We now describe the inverse problem. We first assume that there is only one scatterer $\Omega$ from a priori knowledge. Consider a Jordan curve $S$ in $\Omega e$, which contains $\Omega$ in its interior. We assume that $S$ is sufficiently smooth. We may think of $S$ as an observatory contour, along which the waves are measured. Suppose that the total wave $u_{\infty}$ is obtained at every point on $S$. The inverse scattering problem is to find shape and location of the scatterer $\Omega$ from given $u \infty$ on $S$ for known one or more incident waves $u i$.

The inverse problem in a usual sense is to find the shape of the scatterer $\Omega$ from given far-field pattern $f(\theta)$ for one or more incident plane waves.

Suppose that the far-field patterns can be known exactly. The uniqueness of the solution to the conventional inverse scattering problem is given by a theorem due to Schiffer ( see e.g. Colton and Kress[1983], Theorem 6.10), stating that $\Omega$ is uniquely determined by a knowledge of $f(\theta)$ on some line element of the unit circle $\left(\theta_{0} \leq \theta \leq \theta_{1}\right)$ for one incident wave $u i$ and for any interval of positive wave numbers $k\left(0<k_{0} \leq k \leq k_{1}\right)$.

We notice that the far-field pattern $f(z)$ regarded as a complex-valued function of the complex numbers $z$ is an entire function ( see Colton and Kress[1983] ). From the knowledge of $f$ on a line element of the unit circle, we can determine $f$ on the entire circle by analytic continuation. Moreover, it is sufficient to know $f$ only on a set of infinitely many number of points on a unit circle, which has at least an accumulating point, in order to determine $f$ on the entire unit circle by the unicity theorem.

For the question of uniqueness of the inverse problem, we can also refer to Kirsch and Kress[1987b], mentioning that $\Omega$ is determined by $f(\theta)$ for some finite number of $u i$ of the same wave number $k$ with different incoming directions.

The far-field pattern $f(\theta)$ depends continuously on the shape of $\Omega$, provided the surface is of $C^{2}$-class. However, we notice that $\Omega$ does not depend continuously on $f(\theta)$. Moreover, in practice the far-field pattern is only determined from measurements inherently subject to a certain amount of experimental error. The available far-field pattern may not belong to the class of functions ( entire functions of exponential type ) corresponding to the direct scattering problem. In this case, no solution exists to our inverse scattering problem. We must also suffer from the lack of information in the observation. The far-field pattern is usually measured at a few selected points. To restore the far-field pattern on the entire unit circle, the Legendre polynomial interpolation may be applied as numerical continuation of analytic functions ( see John[1967] ). We shall apply the Backus-Gilbert method for the restoration in the next section.

\section{The Numerical Optimization Method}

We shall confine the geometry of $\Gamma$ to a star-like curve: $r=\phi(\theta), \quad 0 \leq \theta \leq 2 \pi$ with the periodic real positive single-valued function $\phi$ of $C^{2}$-class with the period $2 \pi$. We assume that for a given $\epsilon>0$, there exists $N(\epsilon)$ such that $\phi$ is uniformly approximated by an $N$-parameter function $\psi$ as $\mid \phi(\theta)-\psi\left(\theta ; c_{1}, c_{2}\right.$, $\ldots, c N) \mid \leq \epsilon$. To this purpose, we may think of the Fourier series approximation of $\phi$, as the Fourier series of such functions converge uniformly.

3.1 Exact data. We assume that, for a given incident wave $u i$, the exact far-field pattern $f(\theta)$ are known on an entire unit circle. To make our problem specific, let $\Omega$ be a soft obstacle . Corresponding 
boundary condition on the surface $\Gamma$ of $\Omega$ is the Dirichlet condition (5). For a hard obstacle, the method can be developed in a similar way. Our inverse scattering problem is to find $\Gamma$ from the knowledge of $f(\theta)$.

The method of solution is iterative and two-fold ( solution of direct problem plus mathematical minimization using homotopy method ) nonlinear parameter optimization. It will be safe to first assume that an initial guess $\Gamma(0)$ to $\Gamma$ is known. For $n=0,1,2, \ldots$, we consider the following process:

(i). Solve the boundary integral equation of the first kind

$$
G q^{(n)}(\xi)=\int_{\Gamma^{(n)}} q^{(n)}(x) G(x, \xi) d \Gamma(x)=u^{i}(\xi), \quad \xi \in \Gamma^{(n)}
$$

for unknown $q(n)(x), \quad x \in \Gamma(n)$.

(ii). Calculate corresponding far-field pattern $f(n)(\theta), 0 \leq \theta<2 \pi$ using (1), (11) and (14).

If $f(n)(\theta)$ coincides with the given $f(\theta)$, then $\Gamma(n)$ is a required solution of our inverse scattering problem. Otherwise, $\Gamma(n)$ must be modified to $\Gamma(n+1)$ so that $f(n)(\theta)$ approaches nearer to the given $f(\theta)$ in some sense and the above process (i) and (ii) is continued for next $n$.

The integral operator $G$ is deduced from the single-layer acoustic potential. We notice that the equation (16) is uniquely solvable in $C(\Gamma(n))$ if and only if the wave number $k$ is not an interior Dirichlet eigenvalue (Colton and Kress[1983]). Since the equation is a Fredholm integral equation of the first kind, the numerical solution is likely to stain by computational errors. To circumvent the difficulty, we shall consider the operator $G$ in $L^{2}$ and apply the Tikhonov regularization: Minimize the functional

$$
\left\|\int_{\Gamma^{(n)}} q^{(n)} G d \Gamma-u^{i}\right\|_{L^{2}\left(\Gamma^{(n)}\right)}+\alpha\left\|q^{(n)}\right\|_{L^{2}\left(\Gamma^{(n)}\right)}
$$

with respect to $q(n)$ with the regularization parameter $\alpha$. Let the minimum be denoted by $q \alpha(n)$. It is given as the solution of the linear equation

$$
\left(G^{*} G+\alpha I\right) q_{\alpha}^{(n)}=G^{*} u^{i}
$$

where $G^{*}: L^{2}(\Gamma(n)) \rightarrow L^{2}(\Gamma(n))$ is the adjoint of $G$.

We shall have some remarks. If we consider a hard obstacle, the corresponding integral operator will be associated with the double-layer acoustic potential and it is a compact operator: $C(\Gamma(n)) \rightarrow C(\Gamma(n))$. From the Riesz-Fredholm theory we can see that the equation is uniquely solvable in $C(\Gamma(n))$ if and only if $k$ is not an interior Dirichlet eigenvalue. If $\Gamma(n)$ has corners, the integral operator fails to be compact in general. Under an assumption on the geometry of corners, which may not hinder engineering applications, Kleinman and Wendland[1977] presented the Fredholm theory for the integral equation of the second kind.

The algorithm is not complete, unless some way of improvement from $\Gamma(n)$ to $\Gamma(n+1)$ is specified. Our plan is the reduction to the nonlinear minimization problem

$$
\underset{c_{j}^{(n)}}{\min } \int_{0}^{2 \pi}\left|f^{(n)}(\theta)-f(\theta)\right|^{2} d \theta
$$

This implies that $N$ parameters $c(n)$ j are searched for so that the integral in (15) is to be minimized. The minimization process can be realized by one of the methods in mathematical optimization. Unless $\Gamma(0)$ is sufficiently close to $\Gamma$, the Newton-like methods may not work out well. We shall employ robust methods, e.g., Box's complex algorithm, for constrained minimization for this purpose (Huester and Mize [1973]). 
However, due to our numerical experience the Box's algorithm applied to the inverse problems under consideration often converges to several local minima, depending on the initially assumed shape of the scatterer. To circumvent the drawback and to make sure of the convergence to a minimum corresponding to the exact shape, we introduce a far-field pattern $g(\theta)$ of the unit circle subject to the same incident wave $u i$ and we consider the homotopy

$$
h(\theta, t)=t f(\theta)+(1-t) g(\theta), \quad 0 \leq t \leq 1 .
$$

From the linearity of the set of all far-field patterns of the form (12), we know that $h(\theta, t)$ corresponds to a scattered wave for all such $t$. Instead of (19), we shall consider

$$
\underset{c_{j}^{(n)}(t)}{\min } \int_{0}^{2 \pi}\left|h^{(n)}(\theta, t)-h(\theta, t)\right|^{2} d \theta .
$$

Starting with $t=0$, we determine $c j(n)(t)$ as we increase $t$ up to $t=1$. When $t=1$, the estimated shape with $c j(n)(1)$ is considered to correspond the given far-field pattern $f(\theta)$. The method is called homotopy continuation method ( Kojima[1981]).

3.2 Exact but lack of information. In a practical situation, we have to cope with the lack of observations. We shall assume that the scattered wave is observed only at $M$ distinct locations $\xi_{j}(j=1,2, \ldots$, $M)$. Let $f\left(\theta_{j}\right)$ be the corresponding values of the far-field pattern. We assume that $f\left(\theta_{j}\right)$ are exact. To restore the analytic function $f(\theta)$ for all $\theta$, we consider the following Cauchy integral representation on the unit circle $C:|\zeta|=1$.

$$
\frac{1}{\pi i}(\mathscr{P}) \int_{C} \frac{f(\zeta)}{\zeta-z} d \zeta=f(z), \quad|z|=1
$$

in the sense of Cauchy's principal values. This leads us to the linear moment problem with $M$ equations:

$$
\frac{1}{\pi i}(\Im) \int_{C} \frac{f(\zeta)}{\zeta-2 j} d \zeta=f\left(\theta_{j}\right), \quad e^{i \theta j}=z_{j} /\left|z_{j}\right| .
$$

The problem can be resolved by the Backus-Gilbert method ( see Colton and Kress[1983], Section 7.1) in the form

$$
f_{M}(\theta)=\sum_{j=1}^{M} f\left(\theta_{j}\right) a_{j}(z), \quad z=e^{i \theta}
$$

where the functions $a j(z)$ are given by the expressions

with

$$
a=\left(a_{1}, a_{2}, \ldots, a_{M}\right)^{T}=\frac{1}{g^{T} S^{-1} g} S^{-1} g \text {, }
$$

$$
S_{j k}(z)=\frac{1}{\pi i}(I) \int_{C}(\zeta-z)^{2} \frac{1}{\left(\zeta-z_{j}\right)\left(\zeta-z_{k}\right)} d \zeta=z_{k}+z_{j}-2 z \text {, }
$$

and

$$
g_{k}=\frac{1}{\pi i}(T) \int_{C} \frac{1}{\zeta-z_{k}} d \zeta=1 \text {. }
$$

The algorithm in this case is reduced to the nonlinear minimization problem 


$$
\underset{c_{j}^{(n)}}{\min } \int_{0}^{2 \pi}\left|f^{(n)}(\theta)-f_{M}(\theta)\right|^{2} d \theta
$$

3.3 Fourier components. Instead of the far-field pattern $f(\theta)$, we shall assume that some Fourier components of the far-field pattern are given. To make our problem specific, let the lower $M$ components $c j$ in the Fourier expansion

$$
f(\theta)=\sum_{j=-\infty}^{+\infty} c_{j} e^{i j \theta}, \quad c_{j}=\frac{1}{2 \pi} \int_{0}^{2 \pi} f(\theta) e^{-i j \theta} d \theta
$$

are given. The restoration of $f(\theta)$ can be reduced to the inverse Fourier transform and the minimization in (28) is considered again. Alternatively in an application of the Backus-Gilbert method, we can see

$$
\begin{array}{ll}
S_{j k}= \begin{cases}\frac{1}{i \pi(j+k)}\left(\theta-\pi+\frac{i}{j+k}\right), & j+k \neq 0 \\
\frac{1}{6 \pi}\left(3 \theta^{2}-6 \pi \theta+4 \pi^{2}\right), & j+k=0\end{cases} \\
g_{k}=\delta_{k 0} .
\end{array}
$$

\section{Examples}

We shall present two examples from the direct scattering problem.

4.1 Direct problems. As an incident wave, we consider the plane wave

$$
u^{i}=e^{i k x_{1}}
$$

of the unit amplitude. The exact solution of the direct problem for a circular scatterer with the radius $a$ is presented by King and $\mathrm{Wu}$ [1959] for $k a=3.1$.

We solve the problem numerically by the boundary element collocation method using linear finite element interpolation functions. Figure 1 shows the mesh used in the approximation.
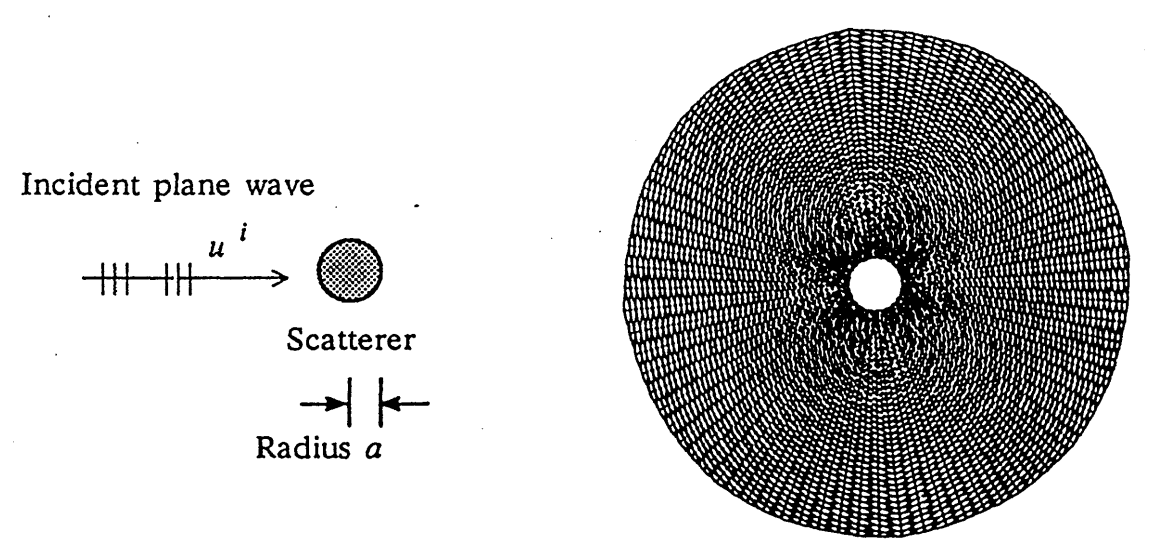

Figure 1. Scattering by a circular cylinder and the mesh.

Suppose for the moment that $\Omega$ is a soft obstacle. Figure 2 shows the calculated contours for the wave components; real, imaginary, absolute and argument of the total wave $u$. 

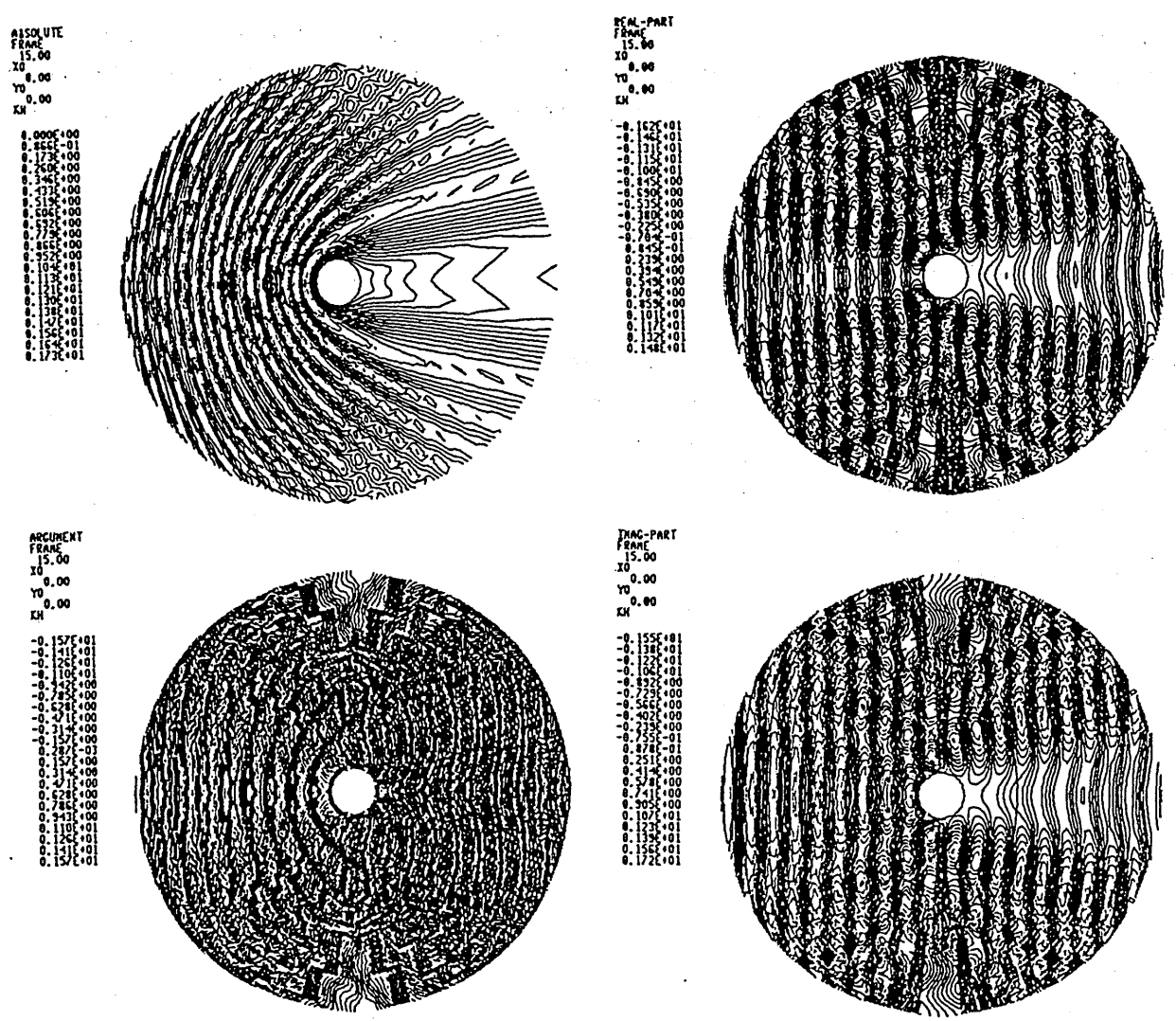

Figure 2. Calculated waves for a soft circular obstacle ( $k a=3.1)$.

We shall assess the sufficient magnitude of $r$ in the asymptotic form (11) when we want $f(\theta)$ from calculated scattered wave us $(x)$. To this purpose, we calculated far-field patterns for different radii $r=$ $10,10^{2}, 10^{3}, 10^{4}$ as the higher order term decreases of the same order of $1 / r$. We know from Figure 3 that $r=10^{3}$ is sufficient for our present purpose.
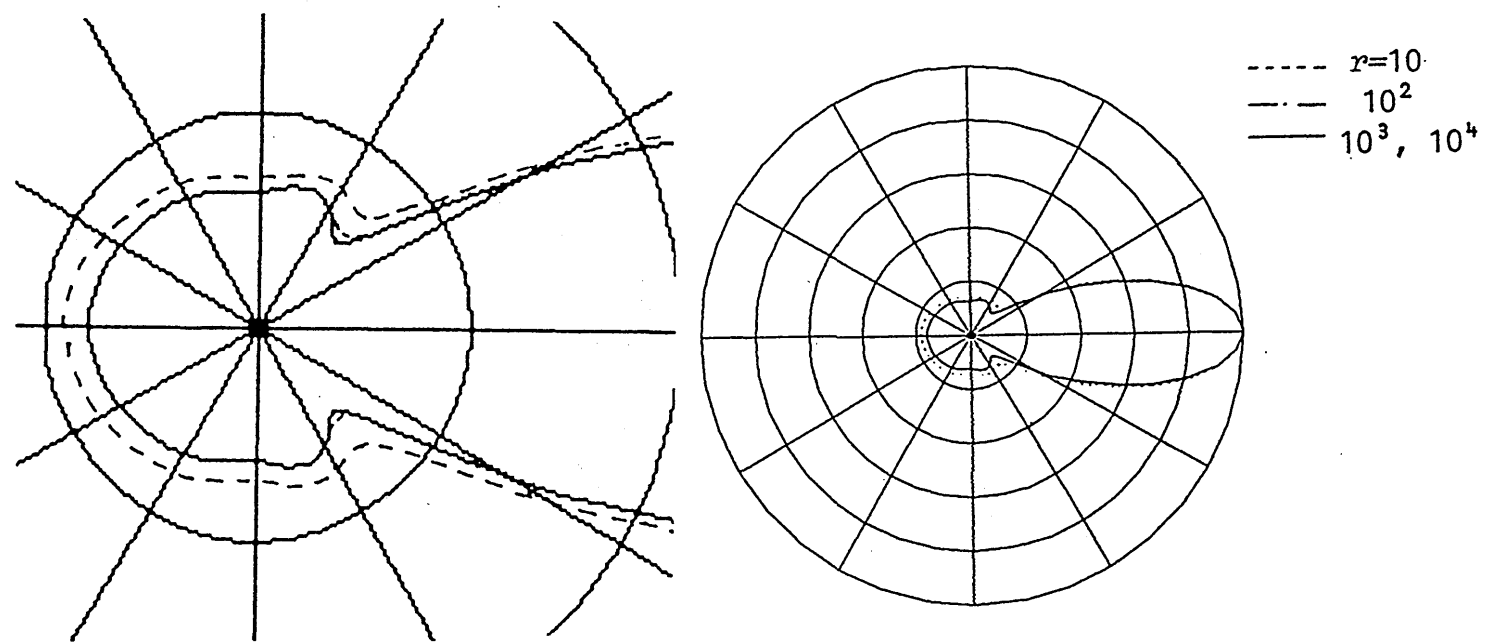

Figure 3. Convergence of scattering cross sections for a unit circular cylinder as $r \rightarrow \infty$. 


\section{6}

Since the equation (15) reduces in this case to the Fredholm integral equation of the first kind, the Tikhonov regularization is applied with the regularization parameter $\alpha=0.1$, which was determined intuitively from our previous experiment . Figure 4 shows the effect of regularization. We can see smoother variations in boundary fluxes.

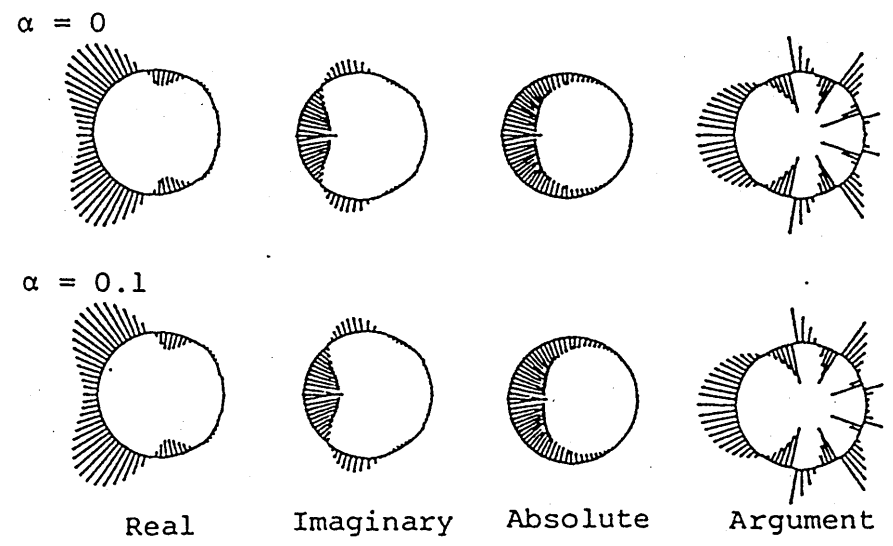

Figure 4. Boundary fluxes.

The exterior Dirichlet problem of the Helmholtz equation (3) is uniquely solvable. However, the boundary integral equation (15) is not uniquely solvable for the interior eigenvalues $k a$ satisfying

$$
J_{n}(k a)=0 \quad(n=0,1,2, \ldots)
$$

with the Bessel functions of order $n$ with a zero $k a=2.40483 \ldots(n=0)$. The direct search for corresponding zero in numerical computation showed that $k a=2.42349$ with the $\log \mid$ determinat of the coefficient matrix $\mid=-122.934$. Figure 5 shows calculated boundary fluxes corresponding to the interior numerical eigenvalue. The calculation was done using single precision arithmatics.

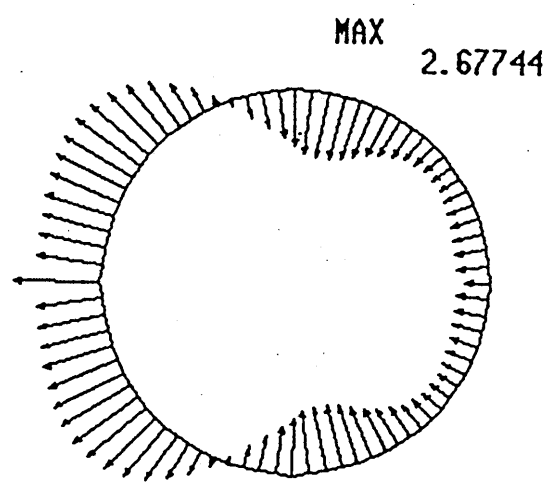

REAL_PART

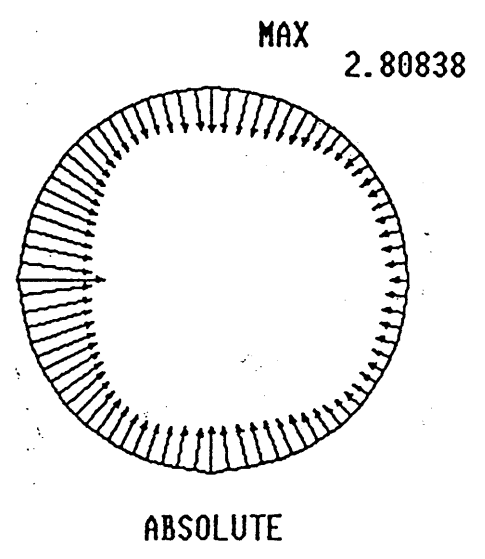

Figure 5. Calculated fluxes with an interior resonant solution ( $k a=2.42349)$. 
Suppose that $\Omega$ is now a hard obstacle. Figure 6 shows the calculated contours of the wave components.
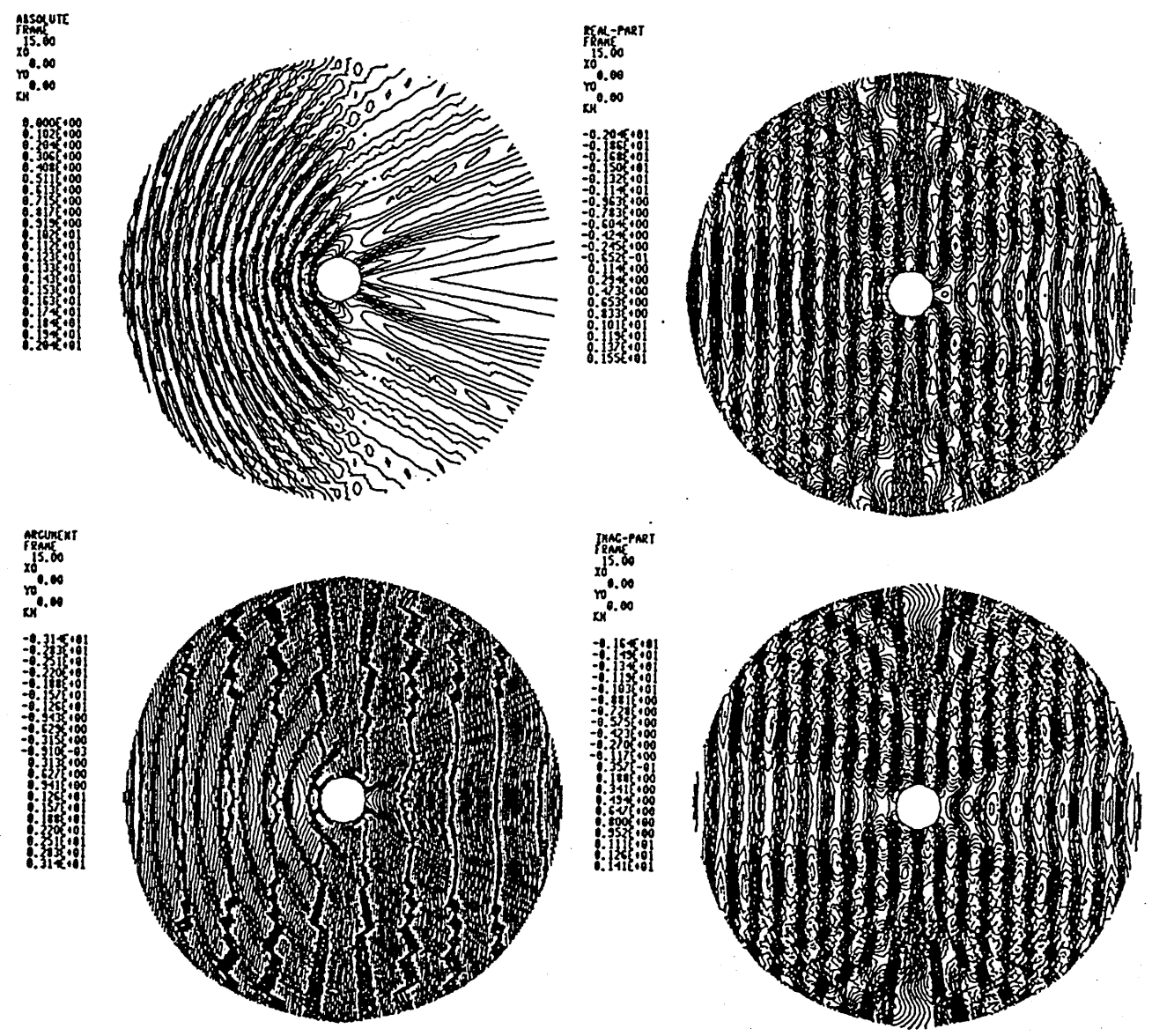

Figure 6. Calculated waves for a hard obstacle $(k a=3.1)$.

The exterior Neumann problem of the Helmholtz equation is uniquely solvable. However, the integral equation (15) is not uniquely solvable for the interior eigenvalues $k a$ satisfying (33). The direct search for corresponding zero in numerical computation shows that $k a=2.40889$ with the $\log \mid$ determinat of the coefficient matrix $\mid=-24.3440$. However, fairly good results can be obtained even for the interior numerical eigenvalue.

4.2 Diffraction. We consider diffraction of the plane wave (32) through a slit as shown in Figure 7. The width $\delta$ of the slit and the wave length $\lambda$ are chosen as $\delta=\lambda=1$. The wave number $k=2 \pi$. 

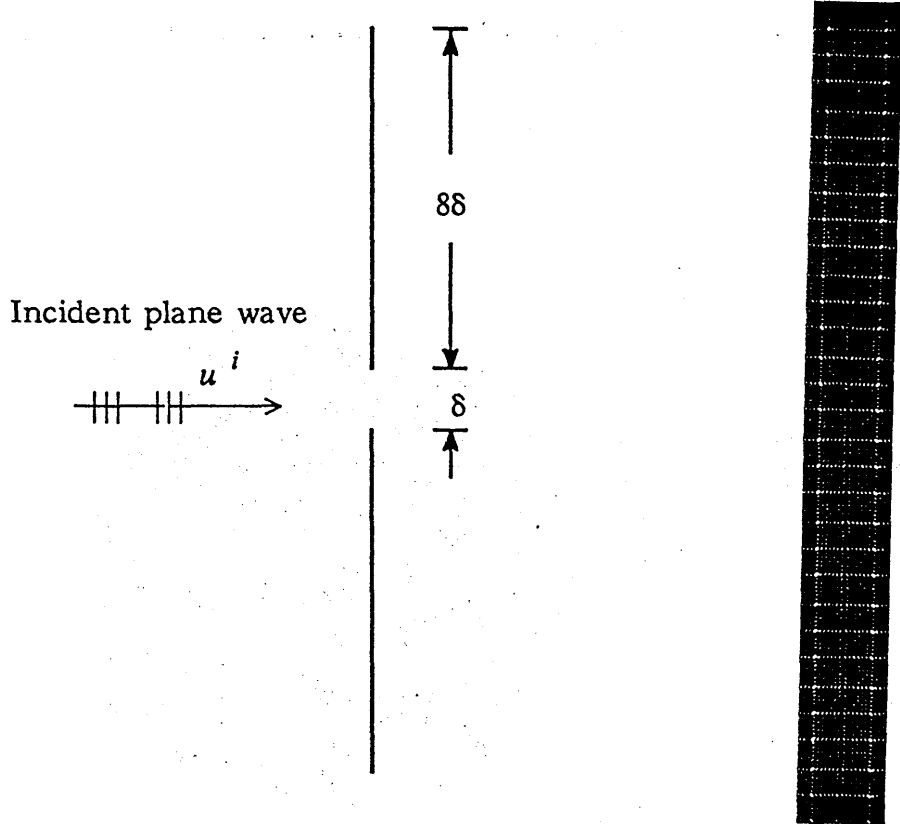

Mesh used

Figure 7. Diffraction of a plane wave through a slit.

Figure 8 shows calculated results. The Fraunhofer diffraction can be seen from the contours of the absolute values of total wave.

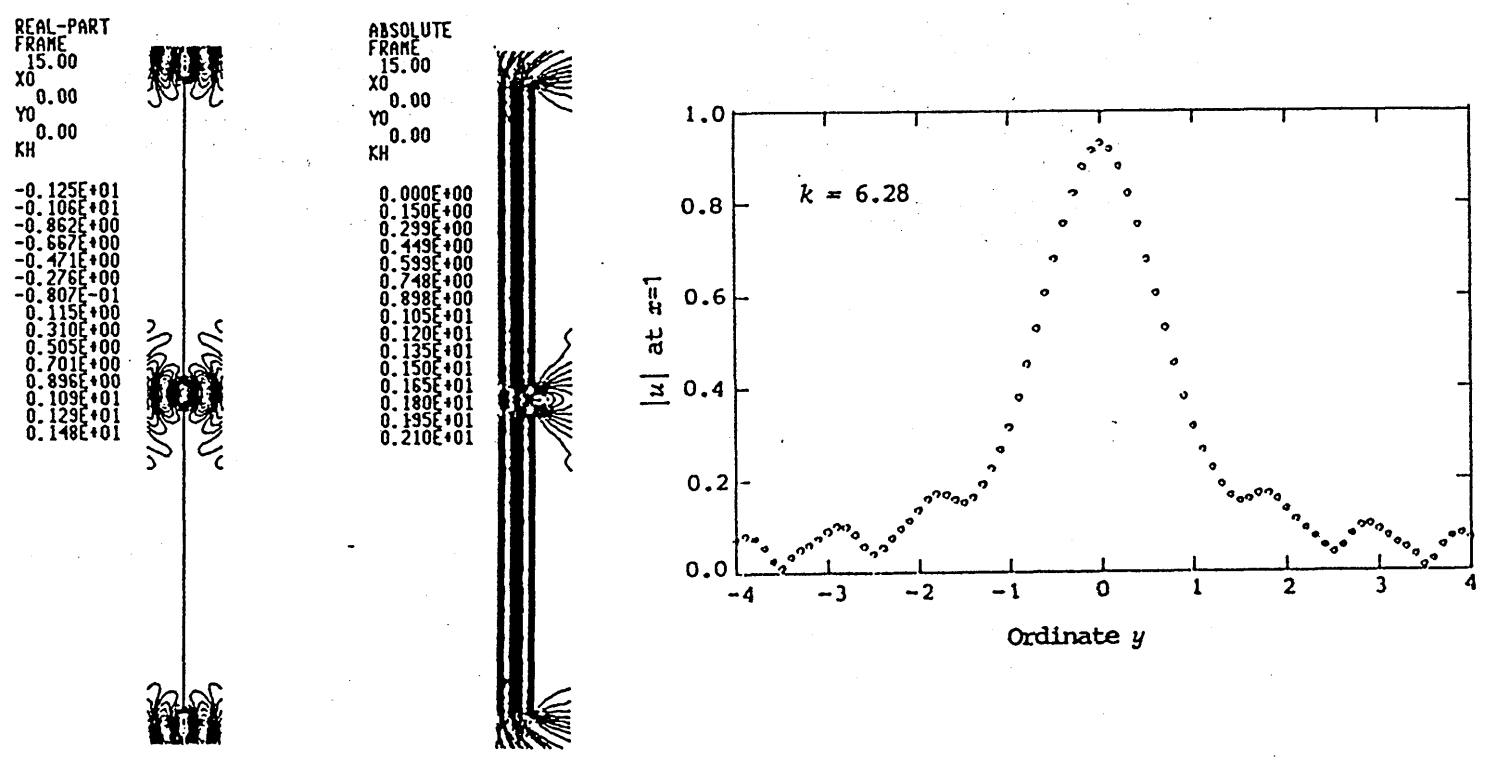

Figure 8. Calculated diffraction by a slit of soft obstacle. 


\subsection{Inverse problems.}

We shall present an inverse problem consisting of finding the shape of an obstacle. We took the complexvalued far-field pattern $f(\theta)$. As the class of functions approximating the shape of cylindrical cross section we assumed

$$
\psi\left(\theta ; c_{1}, c_{2}, \ldots, c_{N}\right)=r_{0}+a_{1} \cos \theta+b_{1} \sin \theta
$$

with unknown parameters $r_{0}, a_{1}, b_{1}$ to be determined.

We shall consider the case when the far-field pattern $f(\theta)$ corresponding to a soft scattering obstacle is known exactly in all directions $0 \leq \theta<2 \pi$. As we mentioned in the last section, no one knows whether the shape of the obstacle can be uniquely determined mathematically unless far-field patterns are given for all wave numbers $k$ contained in some interval $0 \leq k_{1} \leq k \leq k_{2}$. In what follows, we shall study numerically how much the shape can be identified based on the exact knowledge of one far-field pattern corresponding to the plane wave (32) with $k=3.1$.

Box's complex algorithm: Prior to the solution of the inverse problem, we calculated values of the exact far-field pattern $f(\theta j)$ for $\theta j=2 \pi j / 90 \quad(j=1,2, \ldots, 90)$ corresponding to a cylinder with the cross section of the unit circle $r_{0}=1, a_{1}=b_{1}=0$ in the expression (34). The shape was approximated by an equilateral polygon with 40 vertices. The object function to be minimized is

$$
\sqrt{ } \sum_{j=1}^{90}\left|f^{(n)}\left(\theta_{j}\right)-f\left(\theta_{j}\right)\right|^{2} .
$$

We took $(\alpha, \beta, \gamma, \delta)=\left(1.3,10^{\circ}, 5,10^{\circ 5}\right)$ for the parameters involved in the Box's complex algorithm. The set of constraints we set are $0<\psi, 0<r_{0} \leq 2,-0.5 \leq a_{1}, b_{1} \leq 0.5$. Starting with the initial values $r_{0}\left({ }^{0}\right)=1.5, a_{1}\left({ }^{0}\right)=0.2, b_{1}\left({ }^{0}\right)=-0.2$, the minimization process converged unduly with the minimum value 2.406, resulting in the shape $r_{0}\left({ }^{100}\right)=0.555, a_{1}\left({ }^{100}\right)=-0.500 .2, b_{1}\left({ }^{100}\right)=-0.00181$. Process of the estimation is shown in Figure 9. The experiment failed to identify the shape of the scatterer.

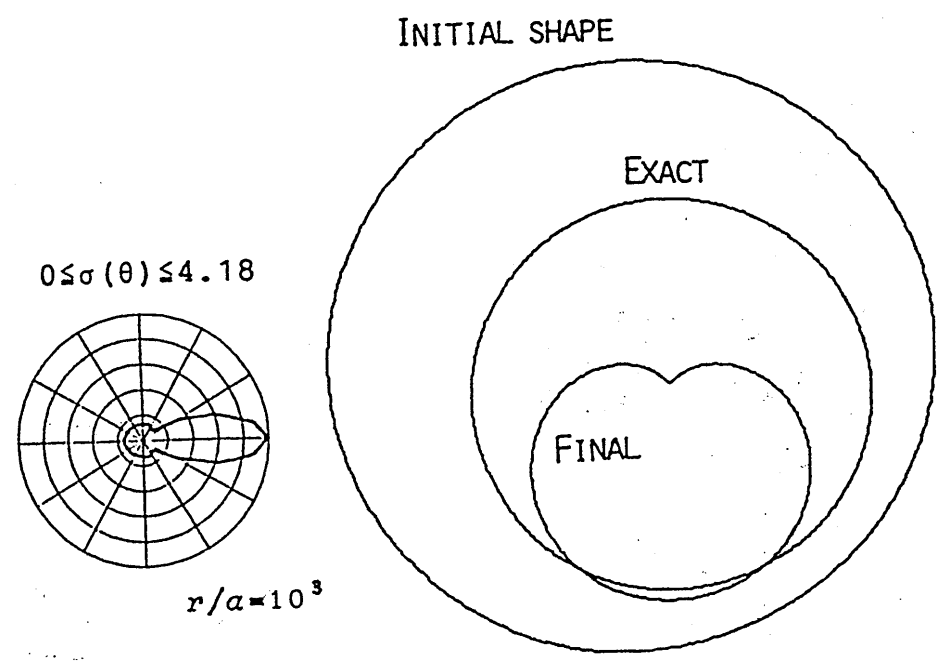

Figure 9. Process of shape identification. 
To examine the reason for the failure, we depicted the surface of the objective function for $0.1 \leq r_{0}$ $\leq 2,-0.5 \leq a_{1}=b_{1} \leq 0.5$ as shown in Figure 10. We can observe that the objective function is not unimodal, has several local minima in particuler in the vicinity of the global minimum.
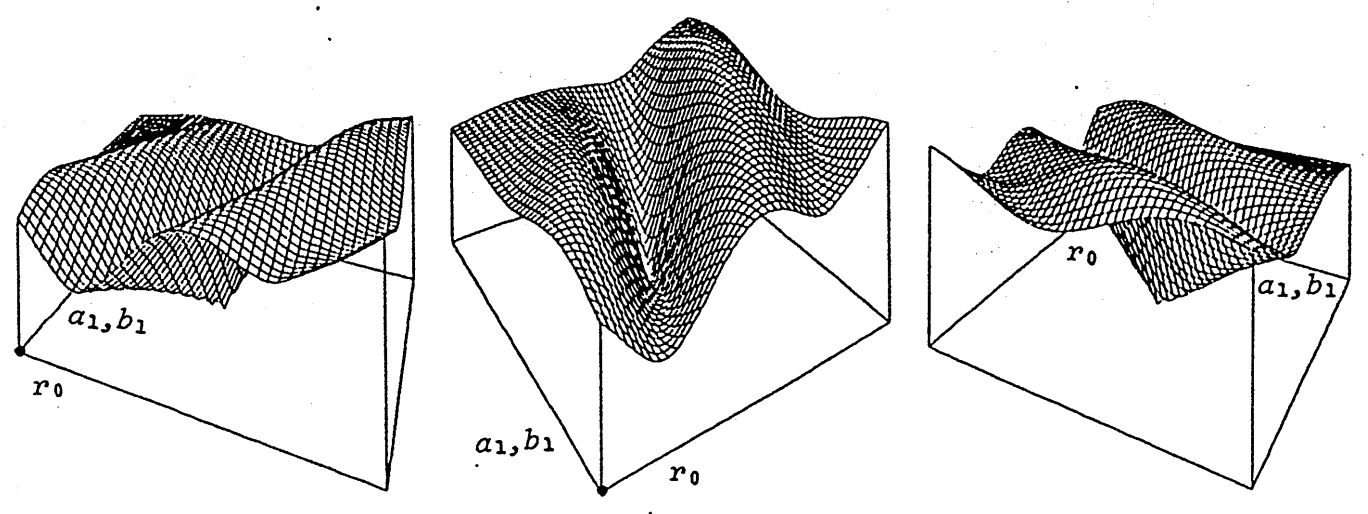

Figure 10. Surface of the objective function.

Homotopy continuation method: We applied the homotopy method to make sure the convergence to the global minimum, independently on the initial shape assumed. For a numerical experiment, we considered a cardiac cylindrical scatterer $r_{0}=0.53840, a_{1}=b_{1}=-0.33333$ as the target. Figure 11 shows the homotopy of far-field patterns and process of identification, starting with the initially assumed unit circular cylinder converging toward the exact shape.
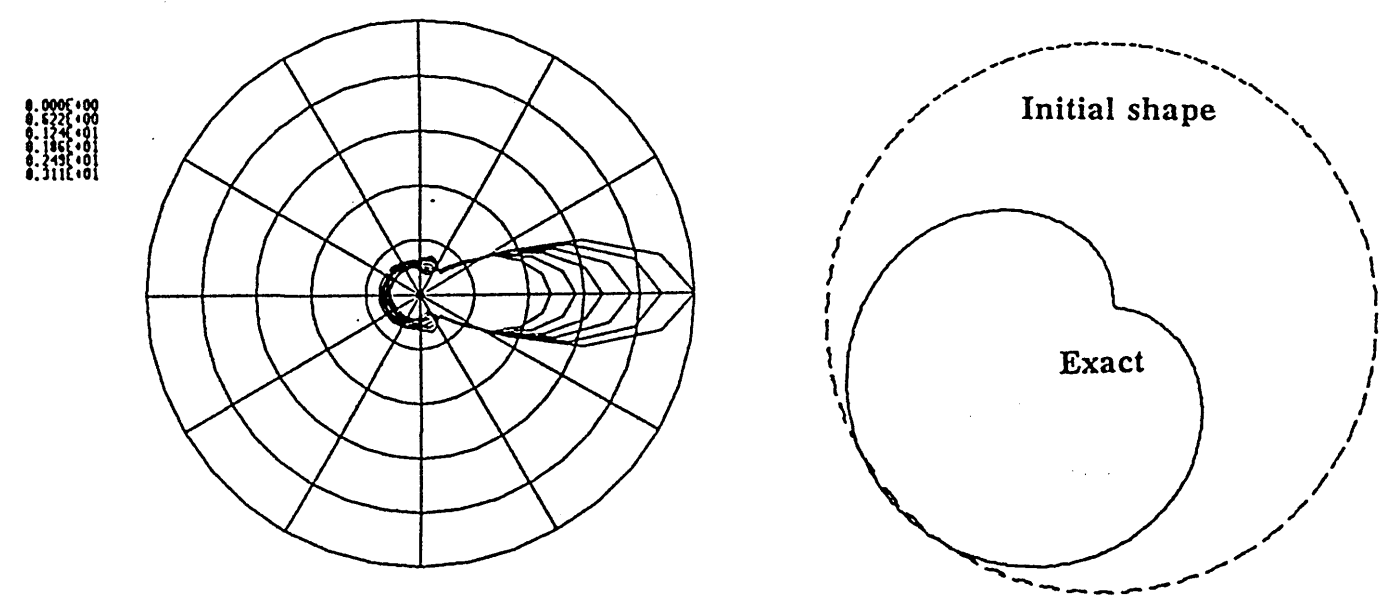

Fugure 11. Identification using homotopy method. 
The numerical process of convergence is summarized in Table 1 . The residual is the value of objective function (35). The final residual corresponding to the homotopy $t=1$ is small. However, residuals corresponding to the intermediate values of $t$ are large, possibly because far-field patterns $h(\theta, t)$ with 0 $<t<1$ do not correspond the shape that can be represented by the expression (34)

Table $1 . \quad$ Homotopy process.

\begin{tabular}{|c|c|c|c|c|c|}
\hline Homotopy No. & \# of iterations & $r_{0}$ & $a_{1}$ & $b_{1}$ & Residual \\
\hline 0 & - & 1 & 0 & 0 & - \\
\hline 1 & 32 & 0.96451 & -0.03139 & -0.17009 & 0.17408 \\
\hline 2 & 31 & 0.92578 & -0.06606 & -0.03724 & 0.32051 \\
\hline 3 & 31 & 0.88193 & -0.10451 & -0.06314 & 0.43542 \\
\hline 4 & 41 & 0.83947 & -0.13915 & -0.09488 & 0.51607 \\
\hline 5 & 29 & 0.75543 & -0.20861 & -0.13200 & 0.52556 \\
\hline 6 & 46 & 0.72182 & -0.22172 & -0.16664 & 0.50036 \\
\hline 7 & 47 & 0.66874 & -0.25766 & -0.20926 & 0.42621 \\
\hline 8 & 27 & 0.62215 & -0.28703 & -0.25094 & 0.31384 \\
\hline 9 & 40 & 0.58133 & -0.30905 & -0.29302 & 0.17010 \\
\hline 10 & 121 & 0.53872 & -0.33295 & -0.33330 & 0.00089 \\
\hline Exact & & 0.53840 & -0.33333 & -0.33333 & \\
\hline
\end{tabular}

N.B. $r_{0}+a_{1} \cos \theta+b_{1} \sin \theta$ with the constraints $0<r_{0} \leq 2,-0.5 \leq a_{1}, b_{1} \leq 0.5$. Box's complex algorithm with $\alpha=1.3, \quad \beta=10^{-4}, \quad y=5, \delta=10^{-4}$.

\section{Concluding Remarks}

We presented some numerical examples in direct and inverse scattering problems. Based on the numerical technique we have together with the formulation proposed in this report, we are going further to develop computational method for the determination of the shape of obstacles.

\section{Acknowledgements.}

Computer implementation is much due to Mrs. Y. Ohura and Mrs. K. Kobayashi.

\section{References}

Colton, D. and R. Kress [1983]: Integral Equation Methods in Scattering Theory. John Wiley \& Sons, Inc., New York.

Groetsch, C. [1984]: The Theory of Tikhonov Regularization for Fredholm Equations of the First Kind. Research Notes in Mathematics 105, Pitman Advanced Publishing Program, Boston.

Hirose S. [1987]: A study on direct and inverse scattering problems of elastic waves. Doctoral Dissertation, Kyoto University. 
Hofmann, B. [1986]: Regularization for Applied Inverse and Ill-Posed problems. Band 85, TeubnerTexte zur Mathematik, Teubner Verlagsgesellschaft, Leipzig.

John, F. [1967]: Lectures on Advanced Numerical Analysis. Gordon and Breach, Science Publishers, Inc., New York.

King, R. W. P. and T. T. Wu [1959]: The Scattering and Diffraction of Waves. Harvard University Press, Cambridge, Massachusetts.

Kirsch, A. and R. Kress [1987a]: A numerical method for an inverse scattering problem. pp.279-289 in H. W. Engl and C. W. Groetsch (Eds.): Inverse and Ill-Posed Problems. Academic Press, Boston.

Kirsch, A. and R. Kress [1987b]: An optimization method in the inverse acoustic scattering. pp.3-18 in C. A. Brebbia, W. L. Wendland, and G. Kuhn (Eds.): Boundary Elements IX, Vol.3, Fluid Flow and Potential Applications. Springer-Verlag, Berlin.

Kleinman, R. E. and W. L. Wendland [1977]: On Neumann's method for the exterior Neumann problem for the Helmholtz equation. Journal of Mathematical Analysis and Applications, pp.170-202, No. 57.

Kojima, M. [1981]: Complementarity and Fixed Points ( in Japanese ). The Series of Mathematical Programming 9, Sangyou-Tosho, Tokyo.

Kress, R. [1989]: Linear Integral Equations. Applied Mathematical Sciences 82, Springer-Verlag, Berlin.

Kuester J. L. and J. H. Mize [1973]: Optimization Techniques. McGraw-Hill, New York.

Landsberga, P. L. and V. A. Borovikova [1982]: Chslennye Metody Teorii Difraktsii ( in Russian ), Mockba, MIP.

Morozov, V. A. [1984]: Methods for Solving Incorrectly Posed Problems. Springer-Verlag, New York. 